\title{
From standard to fractional structural visco- elastodynamics: Application to seismic site response
}

\author{
C. Germoso , A. Fraile, E. Alarcón, J.V. Aguado, F. Chinesta
}

\begin{abstract}
This paper revisits visco-elastodynamics from its most standard formulation to some more advanced description involving frequency dependent damping (or viscosity), analyzing the effects of considering fractional derivatives for representing such viscous contributions. We will prove that such a choice results in richer models that can accommodate different constraints related to the dissipated power, response amplitude and phase angle. Moreover, the use of fractional derivatives allows to accommodate in parallel, within a generalized Kelvin Voigt analog, many dashpots that contribute to increase the modeling flexibility for describing experimental findings. Finally, the effect of fractional damping in dynamic soil models will be addressed within a seismic site analyses framework.
\end{abstract}

Keywords: Elastodynamics, Generalized Kelvin-Voigt model, Fractional derivatives, Seismic site analysis, Soil mechanics

\section{Introduction to standard computational dynamics}

Solid dynamics is usually formulated either in the time or in the frequency domains. The first is preferred when calculating tran sient responses, whereas the frequency approach is an appealing alternative for calculating forced responses, both extensively used and described in many reference books, as for example (Clough and Penzien, 1993). The general discrete form of linear solid dynamics writes

$\mathbf{M} \frac{d^{2} \mathbf{U}(t)}{d t^{2}}+\mathbf{C} \frac{d \mathbf{U}(t)}{d t}+\mathbf{K U}(t) \quad \mathbf{F}(t)$

where $\mathbf{M}, \mathbf{C}$ and $\mathbf{K}$ are respectively the mass, damping and stiffness matrices, $\mathbf{U}$ the vector that contains the nodal displacements and $\mathbf{F}$ the nodal excitations (forces).

The main drawback related to the time integration of Eq. (1) lies in the necessity of solving a linear system (usually of very large size) at each time step, in particular when some of these matrices change in time for a variety of reasons (time dependent behavior, non linearities, ...).
Loads can be easily expressed in the frequency domain. In what follows we consider without loss of generality the simplest sce nario: $\mathbf{F}(t) \quad \mathbf{f} g(t)$, with $\|\mathbf{f}\| \quad 1$. The time function $g(t)$ can be expressed from the superposition of harmonic functions $e^{i \omega t}$, with $\omega$ the circular frequency and $i \quad \sqrt{ }$ 1. If we assume a single frequency harmonic excitation, $g(t) \quad e^{\mathrm{i} \omega t}$, the response of a linear solid is expected having the same frequency but exhibiting a certain phase angle $\theta$, i.e. $\mathbf{U}(t) \quad \overline{\mathbf{U}} e^{\mathrm{i} \omega t+\mathrm{i} \theta}$, where $\overline{\mathbf{U}}$ is the vector containing the amplitude of the nodal displacements. This vector can be rewritten as $\mathbf{U}(t) \quad \overline{\mathbf{U}} e^{\mathrm{i} \omega t+\mathrm{i} \theta} \quad \mathbb{U} e^{\mathrm{i} \omega t}$, where now $\mathbb{U} \quad \overline{\mathbf{U}} e^{\mathrm{i} \theta}$ denotes a vector of complex entries, with $\mathbb{U} \mathbf{U}_{r}+\mathrm{i} \mathbf{U}_{i}$, where $\mathbf{U}_{r}$ and $\mathbf{U}_{i}$ are respec tively the real and imaginary parts of $U$.

By introducing $\mathbf{F}(t) \quad \mathbf{f} e^{\mathrm{i} \omega t}$ and $\mathbf{U}(t) \quad \cup e^{\mathrm{i} \omega t}$ into Eq. (1) it results the frequency based description of solid dynamics

$$
\left(\omega^{2} \mathbf{M}+\mathrm{i} \omega \mathbf{C}+\mathbf{K}\right) \cup \quad \mathbf{f},
$$

where the exponential factor $e^{i \omega t}$ was eliminated from both members.

If damping vanishes, i.e. $\mathbf{C}$, and one focuses on the free response of the mechanical system, i.e. f 0 , then Eq. (2) reduces to: 
$\mathbf{K} \cup \quad \omega^{2} \mathbf{M} \cup$,

that defines an eigenproblem that results in the eigenmodes $\mathbb{U}_{i}$ and the associated eigenfrequencies $\omega_{i}^{2}$. Eigenmode $\mathbb{U}_{i}$ scaled from some normalization condition is called normal mode and is noted by $\phi_{i}$. It is usual to normalize eigenmodes according to $\phi_{i}^{T} \mathbf{M} \phi_{i} \quad M_{i} \quad 1$, from which it results $\phi_{i}^{T} \mathbf{K} \phi_{i} \quad K_{i} \quad \omega_{i}^{2}$, where $M_{i}$ and $K_{i}$ are known as modal mass and modal stiffness respectively. If normal modes are placed in the columns of matrix $\mathbf{P}$, we could express $\mathbb{U}$ in the orthonormal basis defined by the normal modes, according to

$\mathbb{U} \mathbf{P} \cdot \xi(t)$.

Now, by injecting (4) into Eq. (1), premultiplying by the trans pose of $\mathbf{P}$ and taking into account the orthogonality conditions $\phi_{j}^{T} \mathbf{M} \phi_{i} \quad 0$ and $\phi_{j}^{T} \mathbf{K} \phi_{i} \quad 0$ when $i \neq j$, it results

$\mathbf{I} \frac{d^{2} \xi(t)}{d t^{2}}+\mathbf{P}^{T} \mathbf{C P} \frac{d \xi(t)}{d t}+\operatorname{diag}\left(\omega_{i}^{2}\right) \xi(t) \quad \mathbf{P}^{T} \mathbf{F}(t)$

where $\mathbf{I}$ is the unit matrix.

When damping vanishes, $\mathbf{C} \quad 0$, the previous equation reduces to a linear system of uncoupled second order ordinary differential equations.

When damping applies matrix $\mathbf{C} \equiv \mathbf{P}^{\mathrm{T}} \mathbf{C P}$ is not in general diag onal compromising the efficiency of modal analysis. To circumvent this issue different diagonalization procedures have been proposed and widely used. Two usual diagonalization procedures are: (i) diagonalization by model damping that expresses $\mathbf{C} \operatorname{diag}\left(2 \zeta_{i} \omega_{i}\right)$, where $\zeta_{i}$ denotes the damping ratio for the $i$ th natural mode; and (ii) Rayleigh diagonalization that by assuming $\mathbf{C} \quad a_{0} \mathbf{M}+a_{1} \mathbf{K}$ re sults in $\mathbf{C} \operatorname{diag}\left(a_{0}+a_{1} \omega_{i}^{2}\right) \quad \operatorname{diag}\left(2 \zeta_{i} \omega_{i}\right)$, with $\zeta_{i} 1 / 2\left(a_{0} / \omega_{i}+a_{1} \omega_{i}\right)$. These choices imply approximations whose validity and accuracy must be checked.

A more precise route consists of extracting the modes from the solution of the quadratic complex eigenproblem

$$
\left(\mathbf{K}+\mathrm{i} \omega \mathbf{C} \quad \omega^{2} \mathbf{M}\right) \mathbb{U} \quad 0 .
$$

However, many times models involves parametric damping, that is, damping depends on some parameters grouped in vector $\mu$, $\mathbf{C}(\boldsymbol{\mu})$, and in that case the solution of parametric quadratic eigen problems remains an open issue (Quraishi et al., 2014) (Tisseur and Meerbergen, 2001).

When one is interested in solving problems with parametric damping, the best choice, in our opinion, is renouncing to direct time integrations and also to modal analysis based time in tegrations, in favor of an alternative approach, purely harmonic, making use of Eq. (2).

In what follows we assume that the applied load can be written from the superposition of harmonic functions of angular frequency $\omega$

$g(t) \quad \int_{-\infty}^{\infty} \mathcal{G}(\omega) e^{i \omega t} d \omega$

where $\mathcal{G}(\omega)$ represents the content of each harmonic $e^{\mathrm{i} \omega t}$ in $g(t)$. In fact $\mathcal{G}(\omega)$ is the Fourier transform of $g(t)$

$\mathcal{G}(\omega) \equiv \mathcal{F}(g(t)) \quad \int_{-\infty}^{\infty} g(t) e^{-i \omega t} d t$.

In general $\mathcal{G}\left(\omega<\omega^{-}\right) \quad \mathcal{G}\left(\omega>\omega^{+}\right) \approx 0$, that is $g(t) \approx \int_{\omega}^{\omega^{+}} \mathcal{G}(\omega) e^{\mathrm{i} \omega t} d \omega$

that implies that Eq. (2) must be solved for any value of $\omega \in\left[\omega^{-}, \omega^{+}\right]$

$\left(\omega^{2} \mathbf{M}+\mathrm{i} \omega \mathbf{C}+\mathbf{K}\right) \cup(\omega) \quad \mathbf{f}$,

that leads to the parametric solution $\mathbb{U}(\omega)$, that by applying the superposition principle that characterizes linear behaviors leads to the general solution

$\mathbf{U}(t) \quad \int_{\omega}^{\omega^{+}} \mathcal{G}(\omega) \mathbb{U}(\omega) e^{\mathrm{i} \omega t} d \omega$

The main drawback of that approach is the necessity of solving a linear system related to the solution of Eq. (10) for each value of $\omega$ involved in the discrete inverse transform (11), number that in creases with the frequency interval length $\Delta \omega\left|\omega^{+} \quad \omega^{-}\right|$and with the signal resolution. For this reason, modal analysis is much more employed than harmonic analysis.

In the general parametric case, mass, damping and stiffness matrices can depend on a series of parameters grouped in the vector $\boldsymbol{\mu}$, i.e. $\mathbf{M}(\boldsymbol{\mu}), \mathbf{C}(\boldsymbol{\mu})$ and $\mathbf{K}(\boldsymbol{\mu})$, making difficult, as indicated above, the employ of modal analysis that requires the solution of parametric eigenproblems (Quraishi et al., 2014) (Tisseur and Meerbergen, 2001). On the other hand the use of harmonic anal ysis requires solving Eq. (10) for each frequency $\omega$ and each possible choice of the parameters $\boldsymbol{\mu}_{j}, \mathbb{U}\left(\omega ; \boldsymbol{\mu}_{j}\right)$ to finally compute the discrete sum related to

$\mathbf{U}\left(t ; \mu_{j}\right) \quad \int_{\omega}^{\omega^{+}} \mathcal{G}(\omega) \mathbb{U}\left(\omega ; \boldsymbol{\mu}_{j}\right) e^{\mathrm{i} \omega t} d \omega$

for any choice of the parameters $\mu_{j}$.

Thus, if for example we consider two parameters $\boldsymbol{\mu}^{T} \quad\left(\mu_{1}, \mu_{2}\right)$, each one sampled using hundred values, $\mu_{j}$ involves $10^{4}$ samples, i.e. $j \quad 1, \cdots, 10^{4}$. Now, if we assume $10^{4}$ discrete frequencies involved in the reconstruction of $g(t)$, the calculation of the para metric solution $\mathcal{U}\left(\omega_{i} ; \boldsymbol{\mu}_{j}\right)$ requires solving $10^{8}$ linear systems.

The use of the Proper Generalized Decomposition largely considered in our former works (Chinesta et al., 2011, 2010, 2013, 2014), allows solving the parametric model

$$
\left.\omega^{2} \mathbf{M}(\boldsymbol{\mu})+\mathrm{i} \omega \mathbf{C}(\boldsymbol{\mu})+\mathbf{K}(\boldsymbol{\mu})\right) \mathbb{U}(\omega, \boldsymbol{\mu}) \quad \mathbf{f}
$$

by assuming the separated representation

$\mathbb{U}\left(\omega, \mu_{1}, \mu_{2}\right) \approx \sum_{k 1}^{N} \mathbf{Z}_{k} W_{k}(\omega) \cdot \mu_{k}^{1}\left(\mu_{1}\right) \mathscr{M}_{k}^{2}\left(\mu_{2}\right)$,

where $\mathbf{Z}_{k}$ is a vector of nodal displacements and $W_{k}(\omega), \mathscr{\mu}_{k}^{1}\left(\mu_{1}\right)$ and $\mathscr{M}_{k}^{2}\left(\mu_{2}\right)$ are functions that depend on the extra coordinates $\omega, \mu_{1}$ and $\mu_{2}$, respectively. The construction of the separated represen tation (14) implies the solution of a number of linear systems scaling with the number of terms involved in the finite sum, i.e. in the order of $N$ linear systems ( $N$ being in general of few tens).

In soil mechanics (Pecker, 1984) the damping is assumed scaling with the inverse of frequency. The interested reader can refer to (Crandall, 1970) that analyzed the theoretical consequences of assuming a frequency dependent dashpot parameter. In (Crandall, 
1970) it was proved that even if such a choice succeeded to fit experimental data, when coming back to the time space, causality is lost, and then the resulting expressions in the time domain were called non equations. Anyway, it is important to note that even when considering complex nonlinear frequency dependent damping $\mathbf{C}(\omega)$ in Eq. (2) the problem in the frequency domain re mains linear because here the frequency is a model parameter (or a model extra coordinate within the PGD framework).

In this paper we revisits, starting from standard linear dynamics, some relevant and timely questions concerning the use of fractional derivatives for modeling the damping term, as well as the consid eration of a frequency dependent damping and its combination with fractional derivatives.

\section{From simple to fractional damping}

In what follows we consider dynamical systems involving a single degree of freedom, whose model results the scalar counter part of Eq. (1)

$M \frac{d^{2} u(t)}{d t^{2}}+C \frac{d u(t)}{d t}+K u(t) \quad f(t)$.

If the applied force is harmonic, i.e. $f(t) \quad g e^{\mathrm{i} \omega t}$, then $u(t) \quad v e^{\mathrm{i} \omega t}$, with $v$ the complex amplitude, that introduced into Eq. (15) results

$\left(M \omega^{2}+\mathrm{i} \omega C+K\right) v \quad g$

from which the complex amplitude $v$ reads

$$
v \frac{g}{\left(M \omega^{2}+\mathrm{i} \omega C+K\right)} \text {. }
$$

The applied force, characterized by its intensity and frequency, $g$ and $\omega$ respectively, the system mechanical properties $M, C$ and $K$ and the resulting complex amplitude $v$, the last characterized by its intensity $\|v\|$ and the phase angle $\theta, v \quad\|v\| e^{\mathrm{i} \theta} \quad v_{r}+\mathrm{i} v_{i}$, are related by Eq. (17).

Thus, the real and imaginary components of the complex amplitude $v$ read respectively:

$$
v_{r} \quad g \frac{M \omega^{2}+K}{\left(M \omega^{2}+K\right)^{2}+\omega^{2} C^{2}},
$$

and

$$
v_{i} \quad g \frac{\omega C}{\left(M \omega^{2}+K\right)^{2}+\omega^{2} C^{2}},
$$

that leads to the following expressions for the intensity and phase angle

$$
\|v\| \quad \sqrt{ } v_{r}^{2}+v_{i}^{2} \frac{g}{\sqrt{ }\left(M \omega^{2}+K\right)^{2}+\omega^{2} C^{2}},
$$

and

$$
\tan (\theta) \quad \frac{v_{i}}{v_{r}} \quad \frac{\omega C}{M \omega^{2}+K}
$$

If we focus in the expressions given the intensity and the phase angle, Eqs. (20) and (21), we can see that, as soon as $M, C$ and $K$ are given, both depend on the applied frequency:
- If $\omega \rightarrow 0, \tan (\theta) \rightarrow 0$, that is, the force and the response are in phase, like in the case of linear elastic solids, and from Eq. (20) we notice that as expected $\|v\| \approx g / K$.

- If $\omega \rightarrow \infty$, we obtain from Eq. (20) $\|v\| \approx 0$.

- If the applied force and the measured response for different frequencies $\omega$ do not follow the predictions given by Eqs. (20) and (21) the only possibility is assuming a frequency dependance of $M, C$ and/or $K$.

Many authors proposed the use of fractional damping because damping is more difficult to characterize than mass or stiffness. In Appendix we found the definition of fractional derivatives as well as an important property that is used in the sequel, its Fouriers transform.

By considering the fractional damping of power $\alpha$, Eq. (15) reads

$M \frac{d^{2} u(t)}{d t^{2}}+C \frac{d^{\alpha} u(t)}{d t^{\alpha}}+K u(t) \quad f(t)$,

that considering harmonic loading and applying the Fourier's transform (see Appendix) results

$\left(M \omega^{2}+(\mathrm{i} \omega)^{\alpha} C+K\right) v \quad g$.

In the present case, the real and imaginary components of the complex amplitude $v$, taking into account that $i^{\alpha} \cos (\pi / 2 \alpha)+\mathrm{i} \sin \left(\frac{\pi}{2} \alpha\right) \quad p_{r}+\mathrm{i} p_{i}$, read respectively:

$v_{r} \quad g \frac{M \omega^{2}+C p_{r} \omega^{\alpha}+K}{\left(M \omega^{2}+C p_{r} \omega^{\alpha}+K\right)^{2}+\omega^{2 \alpha} p_{i}^{2} C^{2}}$,

and

$v_{i} g \frac{\omega^{\alpha} p_{i} C}{\left(M \omega^{2}+C p_{r} \omega^{\alpha}+K\right)^{2}+\omega^{2 \alpha} p_{i}^{2} C^{2}}$,

that leads to the following expressions for the intensity and phase angle

$\|v\| \quad \sqrt{v_{r}^{2}+v_{i}^{2}} \frac{g}{\left.\sqrt{(} M \omega^{2}+C p_{r} \omega^{\alpha}+K\right)^{2}+\omega^{2 \alpha} C^{2}}$

and

$\tan (\theta) \quad \frac{v_{i}}{v_{r}} \quad \frac{\omega^{\alpha} p_{i} C}{M \omega^{2}+C p_{r} \omega^{\alpha}+K}$.

It can be observed in the expressions given the intensity and the phase angle, Eqs. (26) and (27), that now as soon as $M, C$ and $K$ are given, both depend not only on the applied frequency but also on the damping power $\alpha$. Obviously when taking $\alpha \quad 1$ the expressions reduce to the standard ones and when $\alpha \quad 0$, as expected damping disappears.

Fig. 1 compares the evolution of the phase angle with respect to the frequency $\omega$ and the damping power $\alpha$.

In order to emphasize the physical content of frequency dependent damping and frequency dependent fractional damp ing we are considering a Kelvin Voigt solid characterized by a shear modulus $G$ and viscosity $\eta$.

\section{Viscoelastic solid}

In this section we consider two different variants of the Kelvin Voigt model, one with standard viscosity and the other with frequency dependent viscosity. Then its fractional counterpart will 

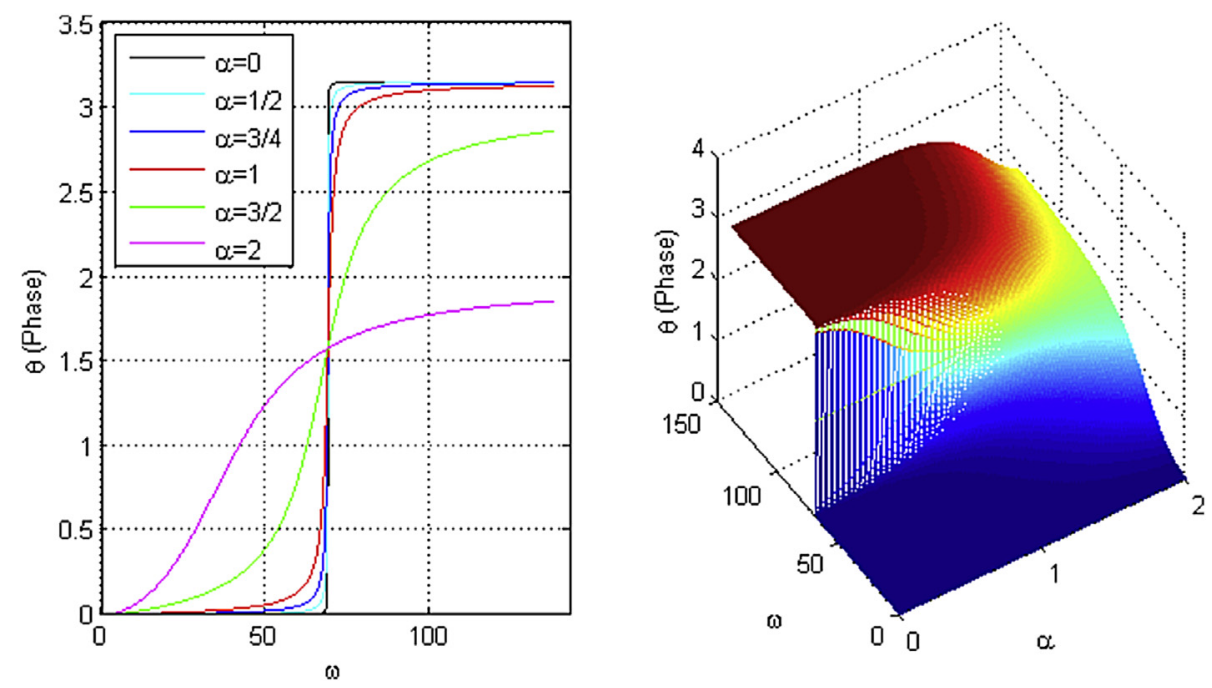

Fig. 1. Phase angle $\theta$ as a function on the frequency $\omega$ and damping power $\alpha, \theta(\omega, \alpha)$.

be addressed for finally defining the generalized Kelvin Voigt model that integrates several viscous elements (dashpots). It is important to notice that such a generalization is only possible in the framework of fractional models as shown in Section 3.3.

\subsection{The standard Kelvin Voigt model}

If as just indicated we define a Kelvin Voigt model by putting in parallel an elastic and a viscous elements, the strains on both ele ments are the same, $\Gamma(t)$, while the total stress $\Sigma(t)$ results from the addition of the elastic and viscous contributions, $\Sigma^{e}(t)$ and $\Sigma^{v}(t)$ respectively. Thus, in the frequency domain assuming again a harmonic stress with amplitude $\tau$, a complex strain response $\gamma$ is expected, both being related by

$\tau \quad \tau^{e}+\tau^{v} \quad G \gamma+\mathrm{i} \eta \omega \gamma \quad(G+\mathrm{i} \eta \omega) \gamma$,

or

$\gamma \frac{\tau}{G+\mathrm{i} \eta \omega}$

whose real and imaginary part results:

$\gamma_{r} \frac{\tau G}{G^{2}+\eta^{2} \omega^{2}}$,

and

$\gamma_{i} \frac{\tau \eta \omega}{G^{2}+\eta^{2} \omega^{2}}$

respectively, that leads to the intensity and phase angle

$\|\gamma\| \quad \frac{\tau}{\sqrt{ } G^{2}+\eta^{2} \omega^{2}}$,

and

$\tan (\theta) \quad \frac{\gamma_{i}}{\gamma_{r}} \quad \frac{\eta}{G} \omega$

From these expressions it can be noticed that:
- If $\omega \rightarrow 0, \tan (\theta) \rightarrow 0$, that is, the force and the response are in phase, like in the case of linear elastic solids, and from Eq. (32) we notice that as expected $\|\gamma\| \approx \tau / G$.

- If $\omega \rightarrow \infty, \tan (\theta) \rightarrow \pi / 2$, that characterizes a linear viscous fluid. Eq. (32) reveals that at very high frequency $\|\gamma\| \approx 0$.

The dissipated power per cycle P results from

$\mathrm{P} \quad \int_{0}^{\frac{2 \pi}{\omega}} \Sigma(t) \frac{d \Gamma(t)}{d t} d t$

with $\quad \Sigma(t) \quad \Re\left(\tau e^{\mathrm{i} \omega t}\right) \quad \tau \cos (\omega t) \quad$ and $\quad \Gamma(t) \quad \Re\left(\gamma e^{\mathrm{i} \omega t}\right) \quad \tau / G^{2}+$ $\eta^{2} \omega^{2}(G \cos (\omega t)+\eta \omega \sin (\omega t))$, that writes

$\mathrm{P} \quad \int_{0}^{\frac{2 \pi}{\omega}} \frac{\tau^{2}}{G^{2}+\eta^{2} \omega^{2}}\left(G \omega \cos (\omega t) \sin (\omega t)+\eta \omega^{2} \cos ^{2}(\omega t)\right) d t$

leading to

P $\frac{\tau^{2} \eta \omega \pi}{G^{2}+\eta^{2} \omega^{2}}$.

Another ways of visualizing the dissipated power in a loading cycle is representing $\Sigma(t)$ versus $\Gamma(t), t \in[0,2 \pi / \omega]$, that as soon as $\eta \neq 0$ exhibits a hysteresis loop whose area represent such a dissi pated power. Fig. 2 depicts the hysteresis loop for four different values of the frequency $\omega 32.42 \mathrm{rad} / \mathrm{s}, \omega 62.83 \mathrm{rad} / \mathrm{s}$, $\omega \quad 94.25 \mathrm{rad} / \mathrm{s}$ and $\omega \quad 125.66 \mathrm{rad} / \mathrm{s}$ when considering $G \quad 2 \cdot 10^{8} \mathrm{~N} /$ $m^{2}, \eta \quad 5 \cdot 10^{6} \mathrm{Ns} / \mathrm{m}^{2}$ and $\tau \quad 1 \mathrm{~N} / \mathrm{m}^{2}$. As it can be noticed the loop area, dissipated power, depends on the considered frequency.

\subsubsection{Frequency dependent viscosity}

In many engineering applications, as for example soil dynamics, it was observed that the dissipated power per cycle does not depend on the applied frequency, that in view of Eq. (36) suggested considering $\eta \quad \zeta / \omega$. As expected, when considering $\eta \quad \zeta / \omega$ the four hysteresis loops coincides as illustrated in Fig. 3. When $\eta \rightarrow 0$ the hysteresis loop approaches to a straight line with negligible area (elastic behavior) and when $G \rightarrow 0$ the loop maximizes its 


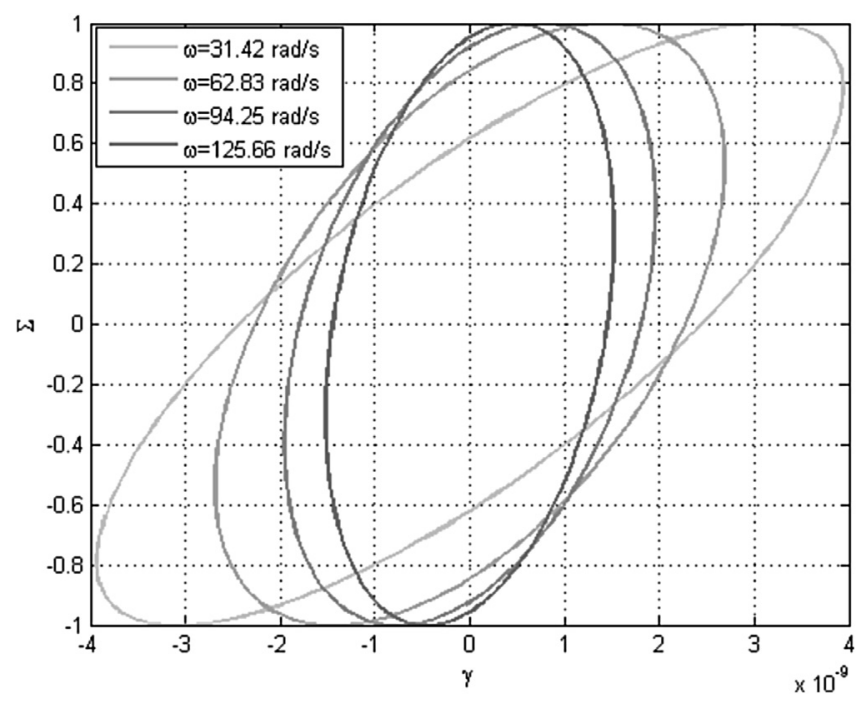

Fig. 2. Hysteresis loops for four different values of the frequency $\omega 32.42 \mathrm{rad} / \mathrm{s}$, $\omega \quad 62.83 \mathrm{rad} / \mathrm{s}, \omega \quad 94.25 \mathrm{rad} / \mathrm{s}$ and $\omega \quad 125.66 \mathrm{rad} / \mathrm{s}$ when considering $G \quad 210^{8} \mathrm{~N} /$ $\mathrm{m}^{2}, \eta \quad 510^{6} \mathrm{Ns} / \mathrm{m}^{2}$ and $\tau \quad 1 \mathrm{~N} / \mathrm{m}^{2}$.

area.

Remark. In soil mechanics it is usual to express $\eta 2 \zeta G / \omega$, however in the sequel we will consider $\eta \quad \zeta / \omega$.

The consideration of a frequency dependent viscosity allows ensuring the constancy of the dissipated power per cycle, however such a choice implies from Eq. (33) the constancy of the phase angle, that now writes $\tan (\theta) \quad \zeta / G$ that becomes independent of the frequency, and from Eq. (32) we conclude on the constancy of the amplitude that now writes $\|\gamma\| \tau / \sqrt{G^{2}+\zeta^{2}}$ and becomes also independent on the applied frequency. All these conditions could be a quite restrictive in some applications, and in those cases
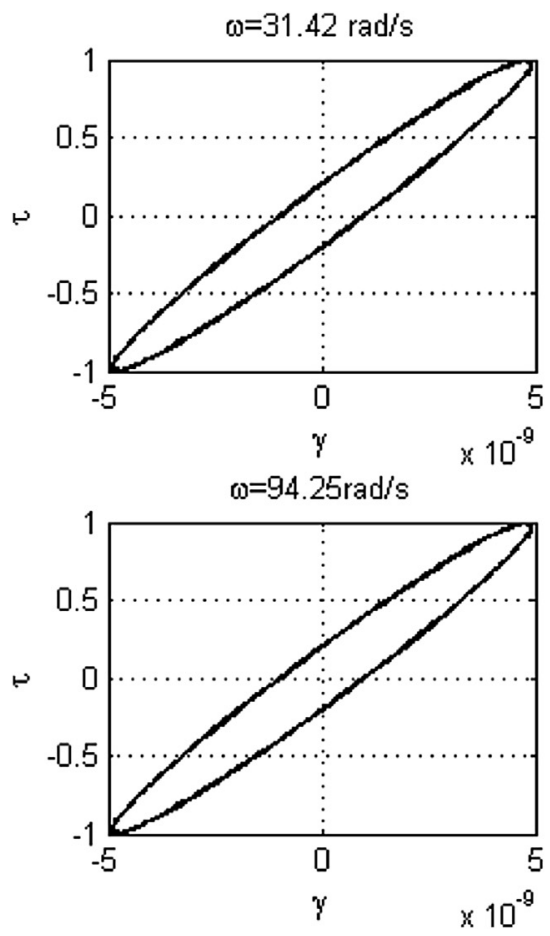

Fig. 3. Hysteresis loops for four different values of the frequency $\omega 32.42 \mathrm{rad} / \mathrm{s}, \omega$ $\eta \quad 510^{6} \mathrm{Ns} / \mathrm{m}^{2}$ and $\tau \quad 1 \mathrm{~N} / \mathrm{m}^{2}$ when considering. $\eta \quad \zeta / \omega$ the consideration of fractional models could relax some of those constraints as discussed in the next section.

\subsection{The fractional Kelvin Voigt model}

The simplest fractional model lies in assuming a fractional viscous behavior. We could also assume a fractional elastic behavior but in the present work and for the sake of simplicity we only as sume fractional derivatives in the term involving the dashpot. Thus the fractional Kelvin Voigt model writes in the frequency domain

$$
\tau \quad \tau^{e}+\tau^{v} \quad G \gamma+\eta(\mathrm{i} \omega)^{\alpha} \gamma \quad\left(G+\mathrm{i}^{\alpha} \eta \omega^{\alpha}\right) \gamma
$$

or

$\gamma \quad \frac{\tau}{G+\mathrm{i}^{\alpha} \eta \omega^{\alpha}}$,

whose real and imaginary parts, considering $\mathrm{i}^{\alpha} \quad p_{r}+\mathrm{i} p_{i}$, results:

$\gamma_{r} \frac{\tau\left(G+p_{r} \eta \omega^{\alpha}\right)}{\left(G+\eta p_{r} \omega^{\alpha}\right)^{2}+\eta^{2} p_{i}^{2} \omega^{2 \alpha}}$

and

$\gamma_{i} \frac{\tau \eta p_{i} \omega^{\alpha}}{\left(G+\eta p_{r} \omega^{\alpha}\right)^{2}+\eta^{2} p_{i}^{2} \omega^{2 \alpha}}$

respectively, that leads to the intensity and phase angle

$\|\gamma\| \quad \frac{\tau}{\sqrt{\left(G+\eta p_{r} \omega^{\alpha}\right)^{2}+\eta^{2} p_{i}^{2} \omega^{2 \alpha}}}$,

and
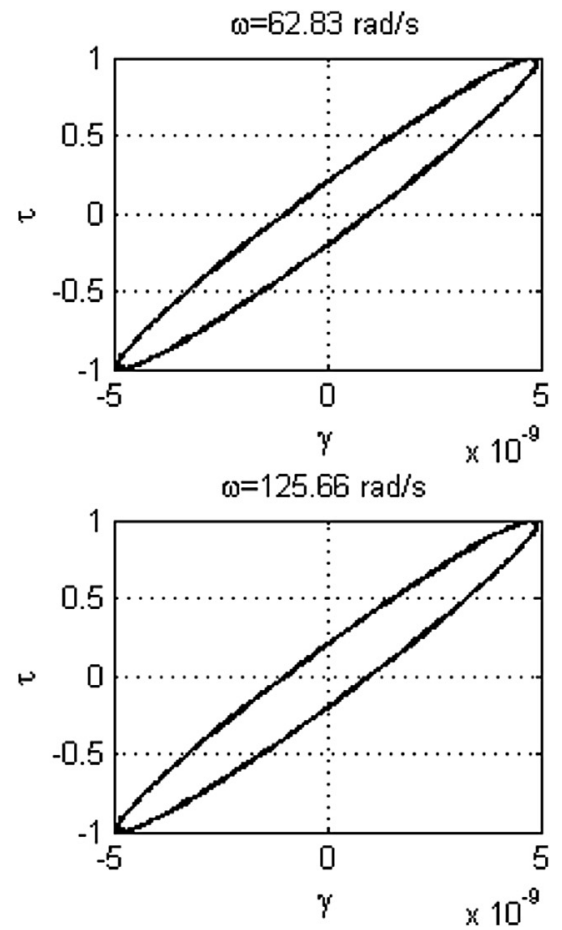

$62.83 \mathrm{rad} / \mathrm{s}, \omega \quad 94.25 \mathrm{rad} / \mathrm{s}$ and $\omega \quad 125.66 \mathrm{rad} / \mathrm{s}$ when considering $G \quad 210^{8} \mathrm{~N} / \mathrm{m}^{2}$, 


$$
\tan (\theta) \quad \frac{\gamma_{i}}{\gamma_{r}} \quad \frac{\eta p_{i} \omega^{\alpha}}{\left(G+p_{r} \eta \omega^{\alpha}\right)}
$$

In order to evidence the hysteric behavior when considering fractional derivatives in the viscous term we consider again a shear

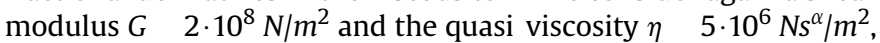
and represents $\Sigma(t)$ versus $\Gamma(t)$ for different $\alpha, \alpha \quad 0.3, \alpha \quad 0.5$, $\alpha \quad 0.8$ and $\alpha \quad 1$ and frequencies $\omega \quad 32.42 \mathrm{rad} / \mathrm{s}, \omega \quad 62.83 \mathrm{rad} / \mathrm{s}$, $\omega \quad 94.25 \mathrm{rad} / \mathrm{s}$ and $\omega \quad 125.66 \mathrm{rad} / \mathrm{s}$. Results are depicted in Fig. 4. In this case it can be observed that the dissipated power per cycle, area of the hysteresis loop, depends on both the frequency $\omega$ and the power $\alpha$. As expected the dissipated power decreases with the power $\alpha$ because when approaching $\alpha \quad 0$ the material becomes the more and more linear elastic.

\subsubsection{Frequency dependent quasi viscosity}

For ensuring that the dissipated power does not depend on the applied frequency, it suffices considering

$$
\eta \frac{\tilde{\zeta}}{\omega^{\alpha}}
$$

that implies

$$
\|\gamma\| \frac{\tau}{\sqrt{\left(G+\tilde{\zeta} p_{r}\right)^{2}+p_{i}^{2}}},
$$

and

$$
\tan (\theta) \quad \frac{\tilde{\zeta} p_{i}}{\left(G+p_{r} \tilde{\zeta}\right)},
$$

where both depend on the power $\alpha$ from $\mathrm{i}^{\alpha} \quad p_{r}+\mathrm{i} p_{i}$.

Fig. 5 depicts for $\eta \quad \zeta / \omega^{\alpha}$ the dissipated power that now does not depends on the frequency but it depends on the dashpot power $\alpha$, decreasing with it as just indicated. It can be noticed that the phase angle also evolves with $\alpha$.
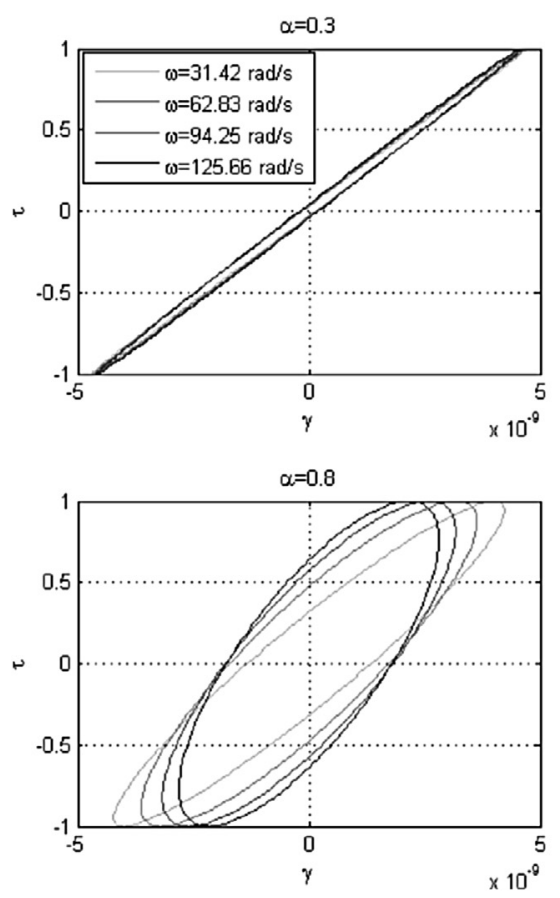

\subsection{The generalized Kelvin Voigt model}

Even if the fractional Kelvin Voigt model offers more flexibility that the standard one, this could be not enough for representing behaviors involving many relaxation times. In that case a valuable route could be considering $\mathcal{V}$ viscous elements, dashpots, with different viscosities $\eta_{1}, \cdots, \eta_{\mathcal{V}}$. If all these elements are disposed in parallel with the linear elastic element the deformation experi enced by all them is the same, $\Gamma(t)$, while the total stress $\Sigma(t)$ results the sum of all contributions, i.e.

$\Sigma \quad \Sigma^{e}+\Sigma^{v, 1}+\cdots+\Sigma^{v, \mathcal{V}}$,

with

$\Sigma^{e} \quad G \Gamma$,

and

$$
\Sigma^{v, i} \quad \eta_{i} \frac{d \Gamma}{d t}, \quad i \quad 1, \cdots, \mathcal{V}
$$

Thus, Eq. (46) results

$$
\Sigma \quad G \Gamma+\eta_{1} \frac{d \Gamma}{d t}+\cdots+\eta_{\mathcal{V}} \frac{d \Gamma}{d t} .
$$

However, Eq. (49) is equivalent to

$$
\Sigma \quad G \Gamma+\left(\eta_{1}+\cdots+\eta_{\mathcal{V}}\right) \frac{d \Gamma}{d t}
$$

that proves that putting all the viscous elements in parallel is equivalent to a single dashpot with viscosity the sum of the $\mathcal{V}$ viscosities. Thus, a behavior composed of viscous elements in par allel has not sense because it does not allow to incorporate the individual behaviors through their respective relaxation times.

However, as soon as we proceed within the fractional frame work the situation becomes radically different. Now each viscous element is associated to a different fractional derivative, defining $\mathcal{V}$
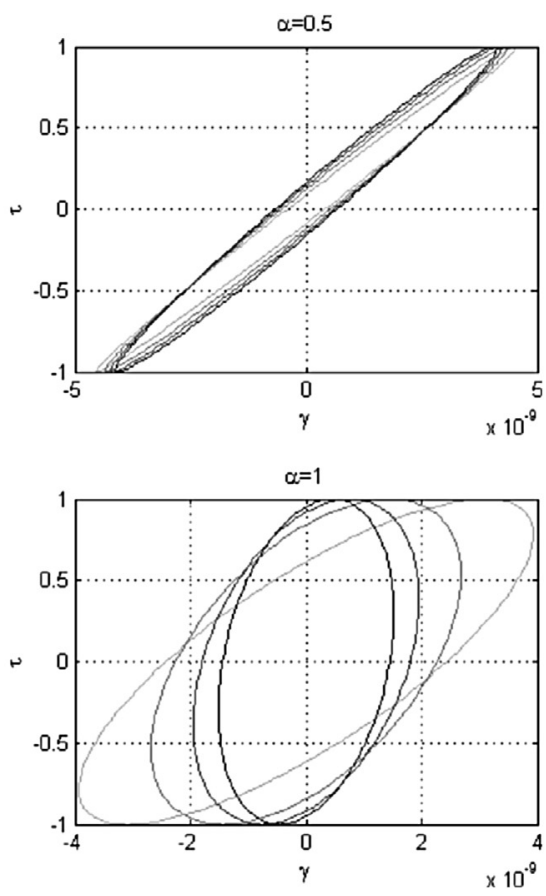

Fig. 4. Hysteresis loop for different $\alpha$ and frequencies $\omega$. 

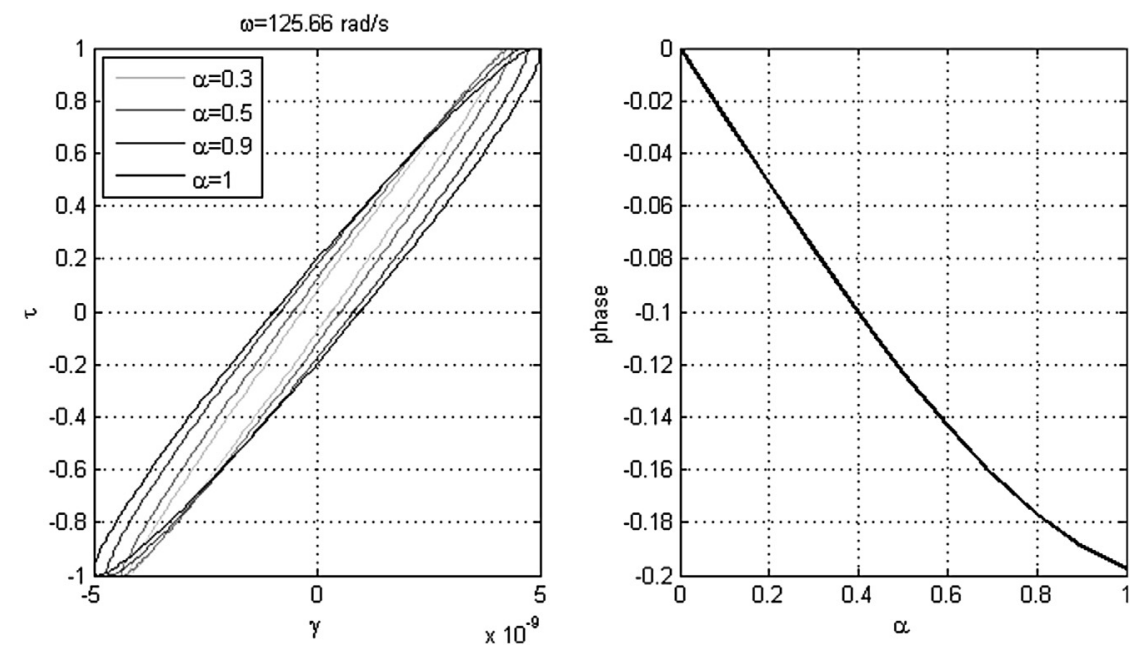

Fig. 5. Hysteresis loop for different $\alpha$ when $\eta \quad \zeta / \omega^{\alpha}$ (left) and evolution of the phase angle with $\alpha$ (right).

couples $\left(\eta_{i}, \alpha_{i}\right), i \quad 1, \cdots, \mathcal{V}$. With the fractional behavior expressed from

$\Sigma^{v, i} \quad \eta_{i} \frac{d^{\alpha_{i}} \Gamma}{d t^{\alpha_{i}}}, \quad i \quad 1, \cdots, \mathcal{V}$,

Eq. (46) reduces to

$\Sigma \quad G \Gamma+\eta_{1} \frac{d^{\alpha_{1}} \Gamma}{d t^{\alpha_{1}}}+\cdots+\eta \eta_{\mathcal{V}} \frac{d^{\alpha_{\mathcal{\nu}}} \Gamma}{d t^{\alpha_{\mathcal{\nu}}}}$,

that cannot be anymore reduced to a single viscous element.

Now, moving to the frequency domain we obtain from Eq. (52)

$\tau \quad G \gamma+\eta_{1}(\mathrm{i} \omega)^{\alpha_{1}} \gamma+\cdots+\eta_{\mathcal{V}}(\mathrm{i} \omega)^{\alpha_{\mathcal{V}}} \gamma$

or

$\tau\left(G+\eta_{1}(\mathrm{i} \omega)^{\alpha_{1}}+\cdots+\eta_{\mathcal{V}}(\mathrm{i} \omega)^{\alpha_{\mathcal{V}}}\right) \gamma$,

that leads to

$\gamma \quad \frac{\tau}{\left(G+\eta_{1}(\mathrm{i} \omega)^{\alpha_{1}}+\cdots+\eta_{\mathcal{V}}(\mathrm{i} \omega)^{\alpha_{\mathcal{V}}}\right)}$,

with $\mathrm{i}^{\alpha_{j}} \quad p_{r}^{j}+\mathrm{i} p_{i}^{j}, j \quad 1, \cdots \mathcal{V}$.

Now, the real and imaginary part of the resulting deformation amplitude $\gamma$ can be extracted from Eq. (55) and with them $\|\gamma\|$ and $\tan (\theta)$.

Fig. 6 compares the evolution of the phase angle with the applied frequency for the standard Kelvin Voigt fractional model KVf - and the generalized Kelvin Voigt fractional model - GKVf the last involving a couple of dashpots. For the KVf fractional model the parameters used are $G \quad 2 \cdot 10^{8} \mathrm{~N} / \mathrm{m}^{2}$ and $\eta \quad 5 \cdot 10^{6} \mathrm{Ns}^{\alpha} / \mathrm{m}^{2}$, with $\alpha \quad 0.5$. In the case of the GKVf model the parameters are $\eta_{1} \quad 5 \cdot 10^{6} \mathrm{Ns}^{\alpha}{ }_{1} / \mathrm{m}^{2}$ and $\eta_{2} \quad 5 \cdot 10^{6} \mathrm{Ns}^{\alpha}{ }_{2} / \mathrm{m}^{2}$, with $\alpha_{1} \quad 0.5$ and $\alpha_{2} \quad 0.8$ respectively. It can be noticed that the consideration of multiple fractional dashpots allows increasing the phase angle with frequency.

\subsubsection{Frequency dependent quasi viscosities}

The choice

$\eta_{i} \quad \frac{\tilde{\zeta}_{i}}{\omega^{\alpha_{i}}}, \quad i \quad 1, \cdots, \mathcal{V}$

allows ensuring the same dissipation per cycle, however $\|\gamma\|$ and $\tan (\theta)$ continue depending on $\alpha_{j}, j \quad 1, \cdots, \mathcal{V}$, from $\mathrm{i}^{\alpha_{j}}$, allowing for a higher model flexibility.

Fig. 7 illustrates the hysteresis loop for fractional Kelvin Voigt models with one and two dashpots, KVf and GKVf models respec tively, for a given frequency $\omega \quad 125.66 \mathrm{~Hz}$. In this example we consider the same rheological parameters that were considered in the previous simulation. It can be observed that the area of the ellipse increases when considering two dashpots, that allows concluding that even if the loop does not evolve with the frequency, the $\alpha$ coefficients allows controlling the dissipated power.

\section{Seismic site analysis}

The equation of linear dynamics in the case of a one dimensional column reads:

$\rho A \frac{\partial^{2} u(z, t)}{\partial t^{2}} \quad G A \frac{\partial^{2} u(z, t)}{\partial z^{2}}$

where $u(z, t)$ is the displacement field, $G$ is the shear modulus, $\rho$ the density and $A$ the area of the column. If a harmonic excitation of frequency $\omega$ applies, the solution of the shear wave propagation equation is also harmonic and of the same frequency, i.e. $u(z, t) \quad \mathcal{U}(z) e^{\mathrm{i} \omega t}$. Thus it results

$$
\rho A \omega^{2} \mathcal{U}(z) \quad G A \frac{\partial^{2} \mathcal{U}(z)}{\partial z^{2}}
$$

When modeling the soil dynamics, the half space is substituted by a viscous damper (Germoso et al., 2015), that when considering a harmonic load of unit amplitude it results

$\left.G A \frac{\partial \mathcal{U}(z)}{\partial z}\right|_{z} \quad 0 \quad c_{s} \rho_{h s} A \dot{\mathcal{U}}_{S} \quad c_{s} \rho_{h s} A \mathrm{i} \omega \mathcal{U}(0)$.

where $\dot{\mathcal{U}}_{S}$ is the measured rock outcropping velocity (Germoso et al., 2015).

On the other hand, the stress free condition at the free surface reads

$\left.G A \frac{\partial U(z)}{\partial z}\right|_{z \quad L} \quad 0$

In the case of simple harmonic motion, damping can conve niently be expressed by using the concept of complex stiffness. The equivalent linear model represents the soil stress strain response 


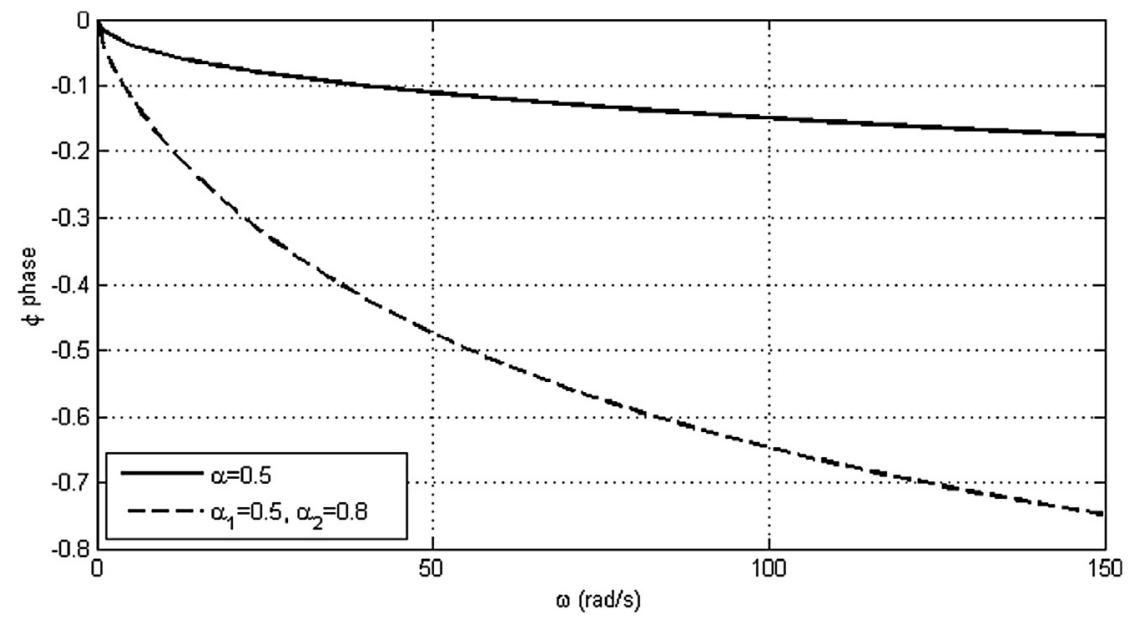

Fig. 6. Comparing the phase angle evolution for the KVf and GKVf models.

from a Kelvin Voigt model. Thus, the shear stress $\tau$ depends on the shear strain $\gamma$ and its rate $\dot{\gamma}$ according to

$\tau \quad G \gamma+G^{\prime} \frac{d \gamma}{d t}$

or in the frequency domain as

$\tau \quad\left(G+\mathrm{i} \omega G^{\prime}\right) \gamma$

The complex shear modulus $G^{*}$ reads

$$
G^{*} \quad G+\mathrm{i} \omega G^{\prime} \quad G(1+\mathrm{i} 2 \zeta)
$$

whose most general case for accommodating fractional damping, writes

$$
G^{*} \quad G+\mathrm{i}^{\alpha} \omega^{\alpha} G^{\prime} \quad G\left(1+\mathrm{i}^{\alpha} 2 \zeta\right)
$$

Observe in Eq. (64) $G$ ' scales with the inverse of frequency in such a manner that the complex shear modulus is now frequency independent. We refer to (Germoso et al., 2015) for a deeper dis cussion on the consequences of this choice. Introducing Eq. (64) into Eq. (58) it results $\rho \omega^{2} \mathcal{U}(z)+G^{*} \frac{\partial^{2} \mathcal{U}(z)}{\partial z^{2}} \quad 0$

whose form, taking into account the just referred boundary con ditions, reads:

$$
\begin{aligned}
\int_{\Omega_{z}} & \mathcal{U}^{*}(z) \rho \omega^{2} \mathcal{U}(z) d z \quad \int_{\Omega_{z}} \frac{\partial \mathcal{U}^{*}}{\partial z} G\left(1+\mathrm{i}^{\alpha} 2 \zeta\right) \frac{\partial \mathcal{U}(z)}{\partial z} d z \\
& \mathcal{U}^{*}(0) \mathrm{i} \rho_{h s} c_{s} \omega \mathcal{U}(0)+\mathcal{U}^{*}(0) \rho_{h s} c_{s} \dot{\mathcal{U}}_{s} \\
& 0
\end{aligned}
$$

where it was assumed that the dashpot representing the half space does not show a fractional behavior. The wave velocity is given by $c_{s} \sqrt{ } G_{h s} / \rho_{h s}$.

\subsection{Nonlinear modeling}

The so called equivalent linear approximation was introduced with the aim of improving constitutive laws of soils. It was imple mented into the EERA (Equivalent linear Earthquake Response Analysis) software (Bradet et al., 2000), a recent implementation of the well known concepts of equivalent linear earthquake site
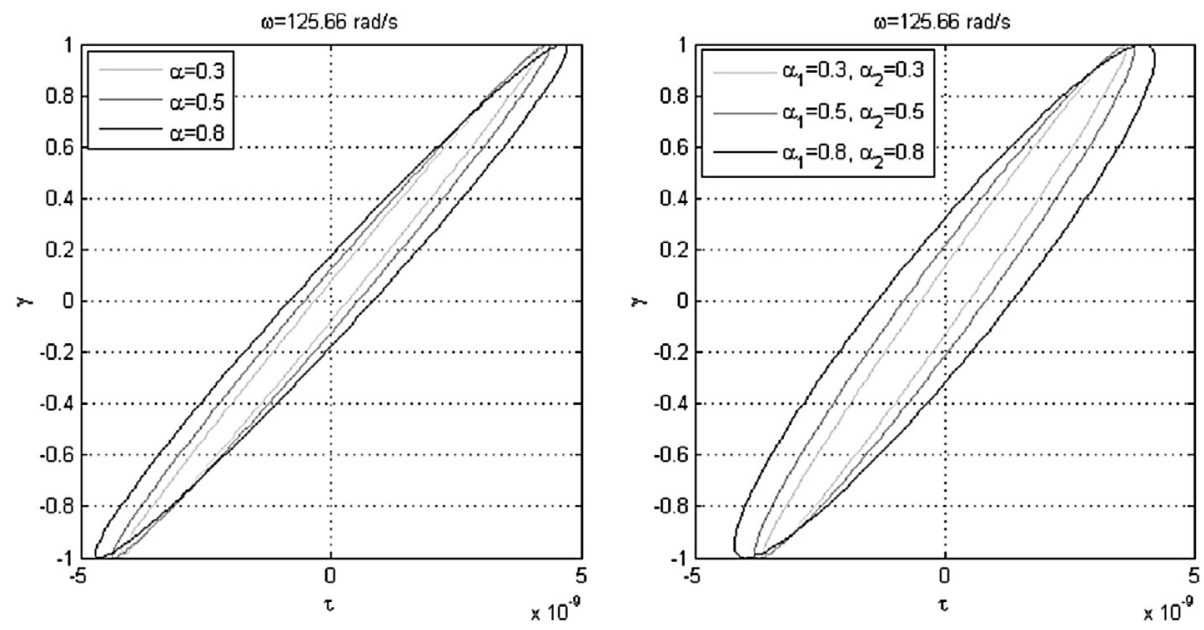

Fig. 7. Hysteresis loop for frequency-dependent viscosities in the case of a KVf (left) and GKVf (right). 

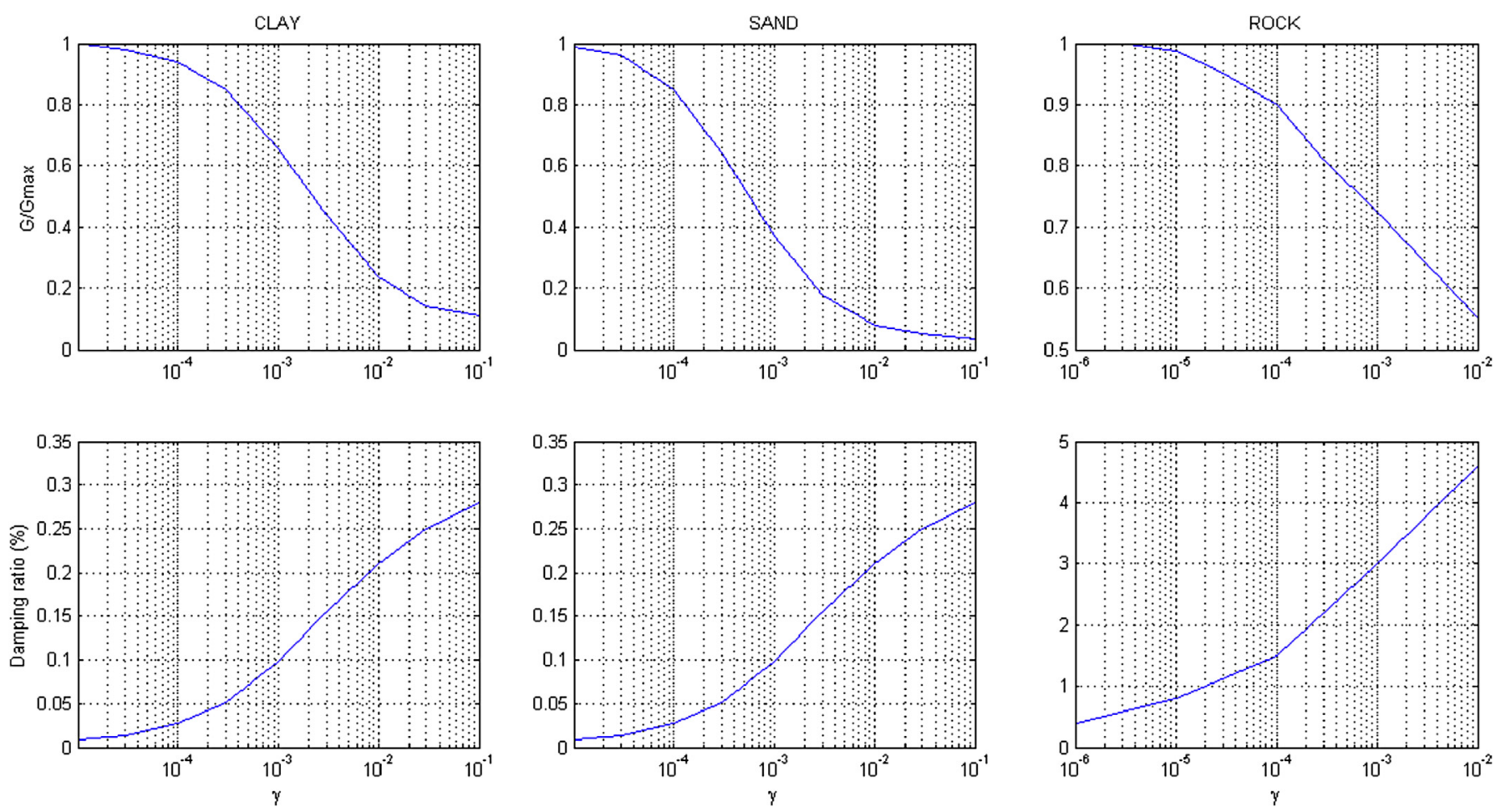

Fig. 8. Normalized shear modulus $\left(G / G_{\max }\right)$ and damping ratio $\zeta$ for sand, clay and rock.

Table 1

Soil properties.

\begin{tabular}{lllccc}
\hline Stratum & Material & Thickness $(\mathrm{m})$ & Wave velocity $(\mathrm{m} / \mathrm{s})$ & Gmax $(\mathrm{MPa})$ & $\zeta(\%)$ \\
\hline 1 & Soft Clay & 15 & 90 & 15 & 11 \\
2 & Clay & 20 & 150 & 40 & 6 \\
3 & Compact sand & 30 & 330 & 200 & 4 \\
4 & Soft rock & 25 & 600 & 700 & 1 \\
5 & Half-space & & 1500 & 4500 & 0 \\
\hline
\end{tabular}

response analysis, starting from the same basic concepts that the SHAKE software (Schnabel et al., Seed). The linear approximation considers the shear modulus $G$ and the damping ratio $\zeta$ constant for each soil stratum and both dependent of the strain level (Bozorgnia
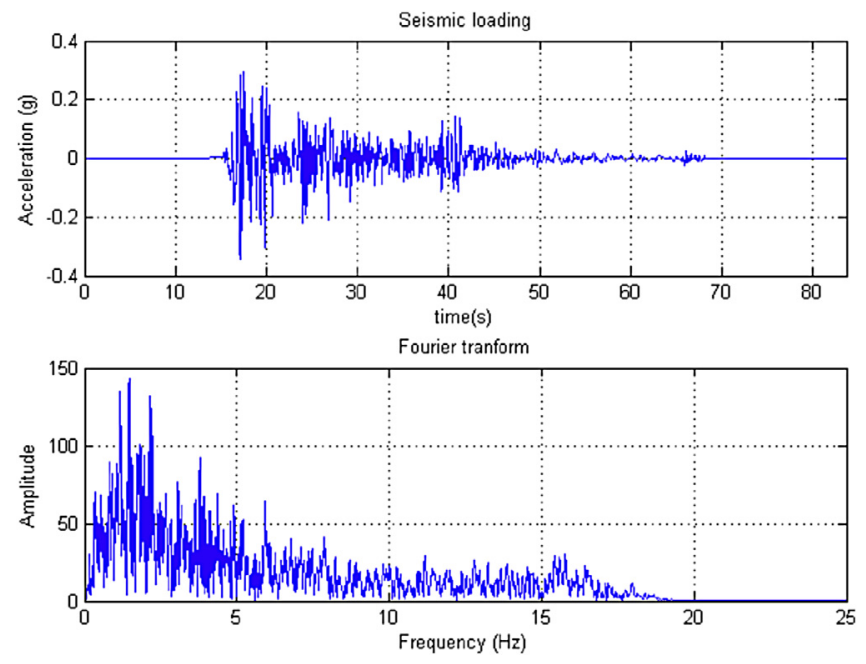

Fig. 9. Acceleration at the bedrock. and Bertero, 2004). Obviously with both parameters depending on the strain, and the last at its turn depending on the parameters choice, the problem becomes nonlinear and an iteration process is required for solving it.

The effective strain is given by $\gamma_{\text {eff }} \gamma \gamma_{\max }$, with $\gamma$ the effective to the maximum shear strains ratio. $\gamma$ depends on earthquake magnitude $M$ and can be estimated from $\gamma \quad M \quad 1 / 10$. An effective strain value of $65 \%$ of the maximum shear strain is usually employed in many applications. In the computations that follow we consider the curves relating the shear modulus and the damping ratio on the effective strain proposed by (Seed and Idriss, 1970), both illustrated in Fig. 8 for sand, clay and rock.

When the strain varies significantly along the deposit deep, or when the soil deposit is composed of different strata of different materials, one must consider different layers to which different values of $G$ and $\zeta$ are assigned depending on the material and the strain level.

In order to speed up calculations, one could compute offline the parametric solution $\mathcal{U}(z, \omega, G, \zeta)$ for the soil deposit, assuming the same values of $G$ and $\zeta$ everywhere in the soil domain. When the soil column is partitioned in $\mathscr{L}$ layers, in which both coefficients are assumed constant, the parametric solution writes $\mathcal{U}\left(z, \omega, G_{1}, \zeta_{1}, \cdots, G_{\mathscr{L}}, \zeta_{\mathscr{L}}\right)$. This route was successfully considered in (Germoso et al., 2015) for the seismic site response. In this section we analyze the impact of fractional damping behavior for a given seismic loading when considering both a linear and nonlinear soil behavior.

\subsection{Seismic loading problem}

In the numerical simulation carried out and discussed below we consider four layers and a half space with the properties in Table 1. In the nonlinear case both the shear modulus and the damping ratio evolves as a function of the effective strain as described above. 
(a)
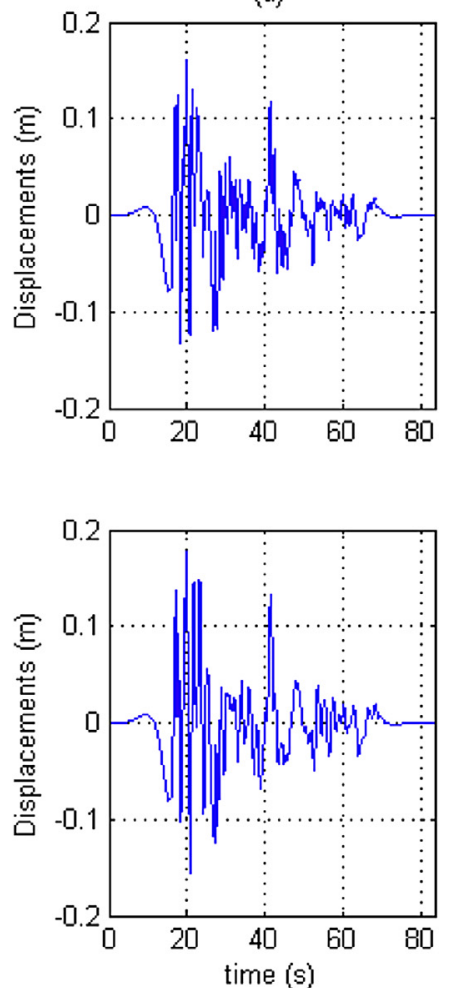

(b)
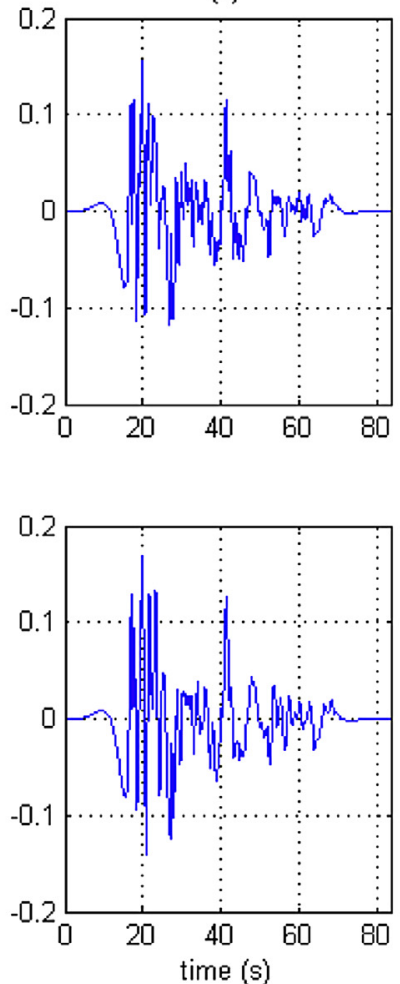

(c)
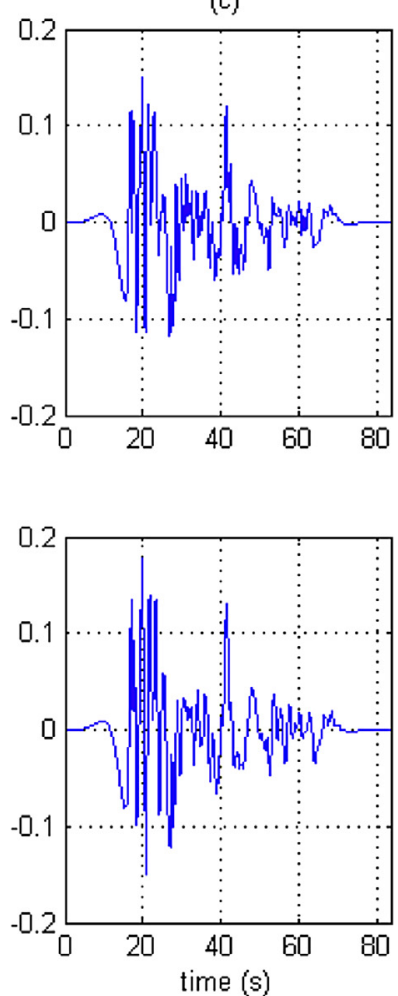

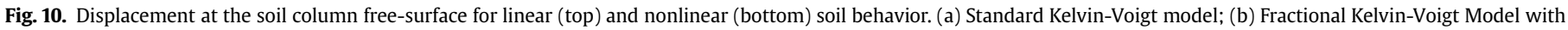
$\begin{array}{lllll}\alpha & 0.1 \text {; (c) Generalized Kelvin-Voigt Model with } \alpha_{1} & 0.1, \alpha_{2} & 0.4 \text { and. } \alpha_{3} & 0.8\end{array}$

(a)
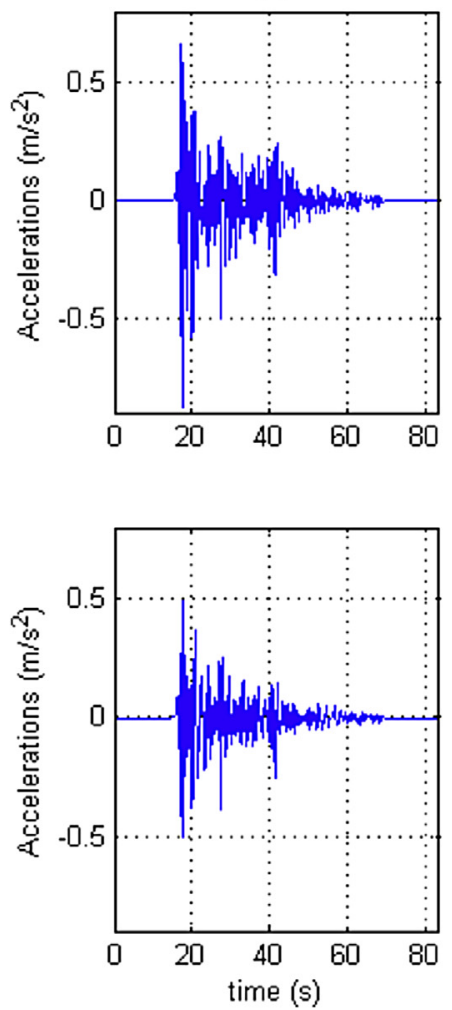

(b)
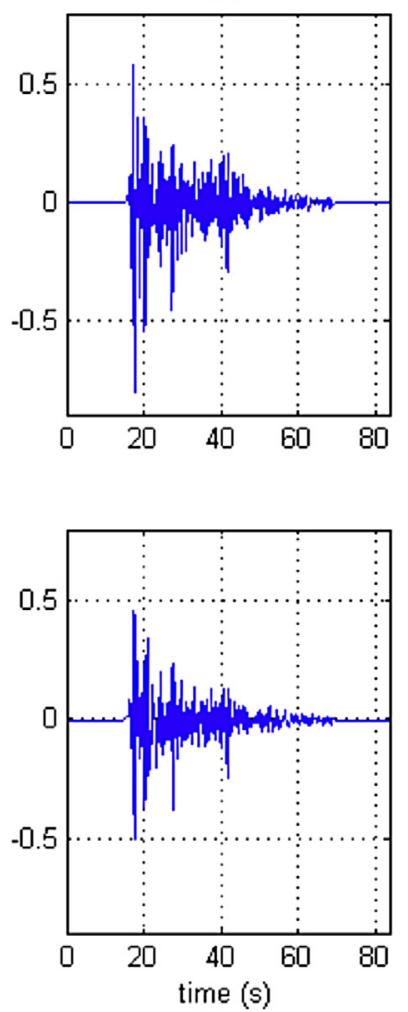

(c)
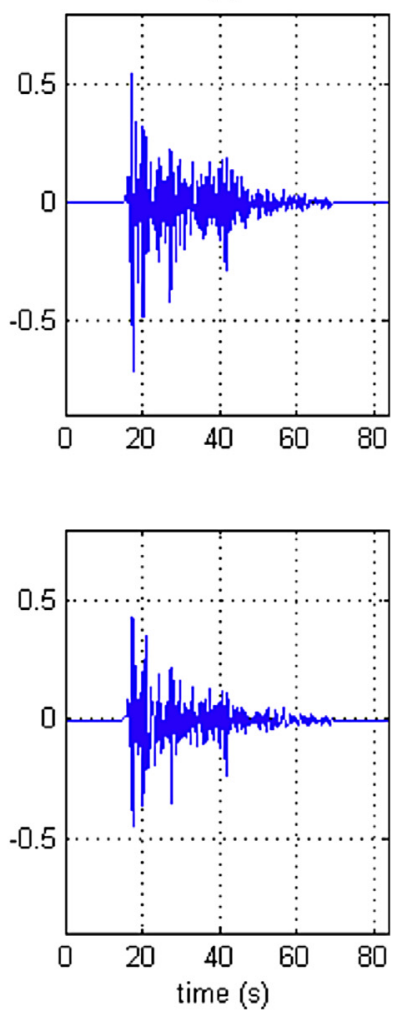

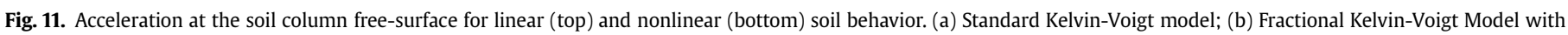

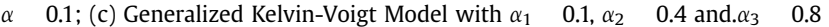



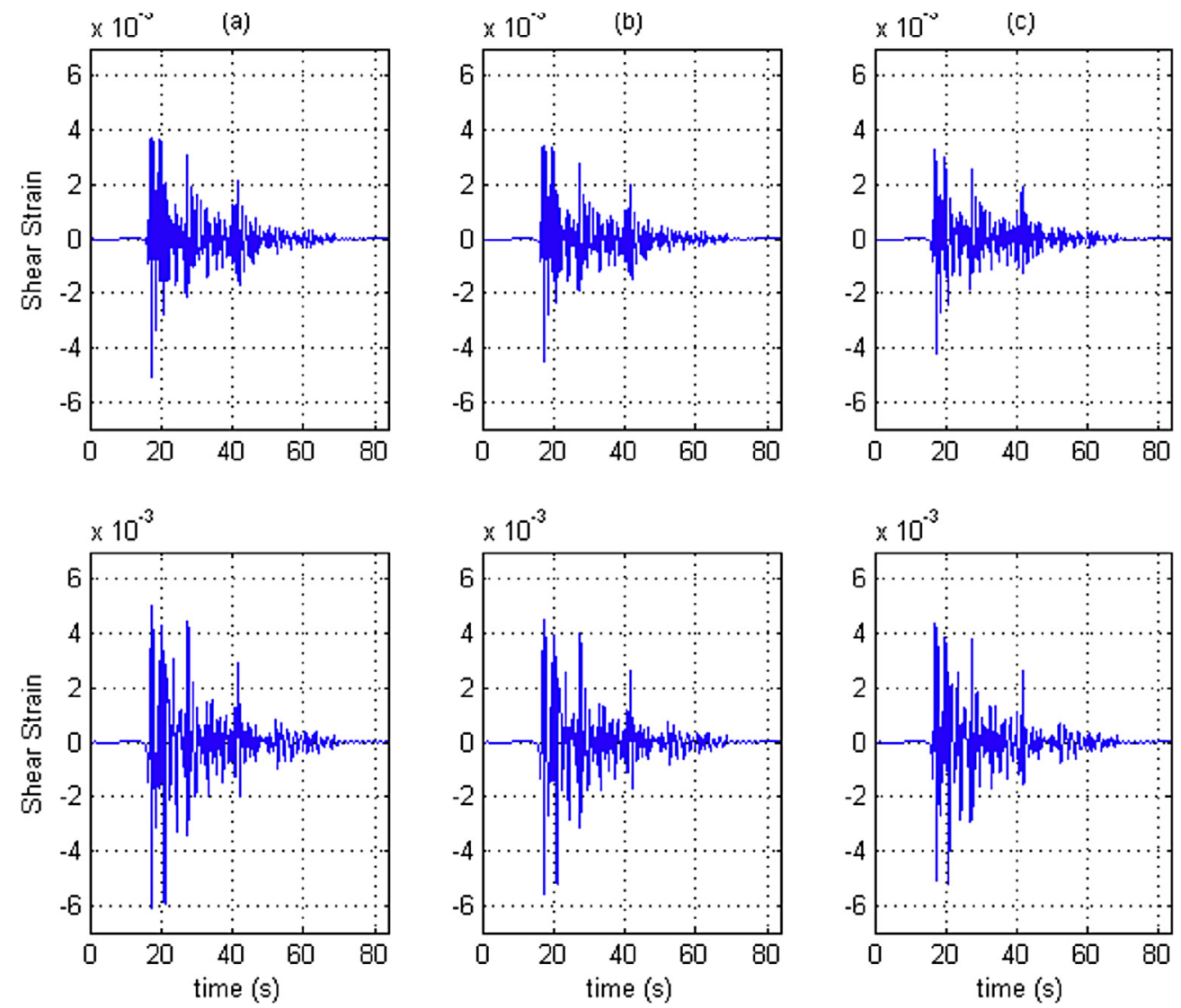

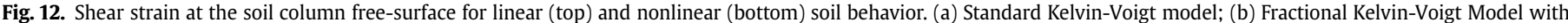
$\begin{array}{lllll}\alpha & 0.1 \text {; (c) Generalized Kelvin-Voigt Model with } \alpha_{1} & 0.1, \alpha_{2} & 0.4 \text { and. } \alpha_{3} & 0.8\end{array}$
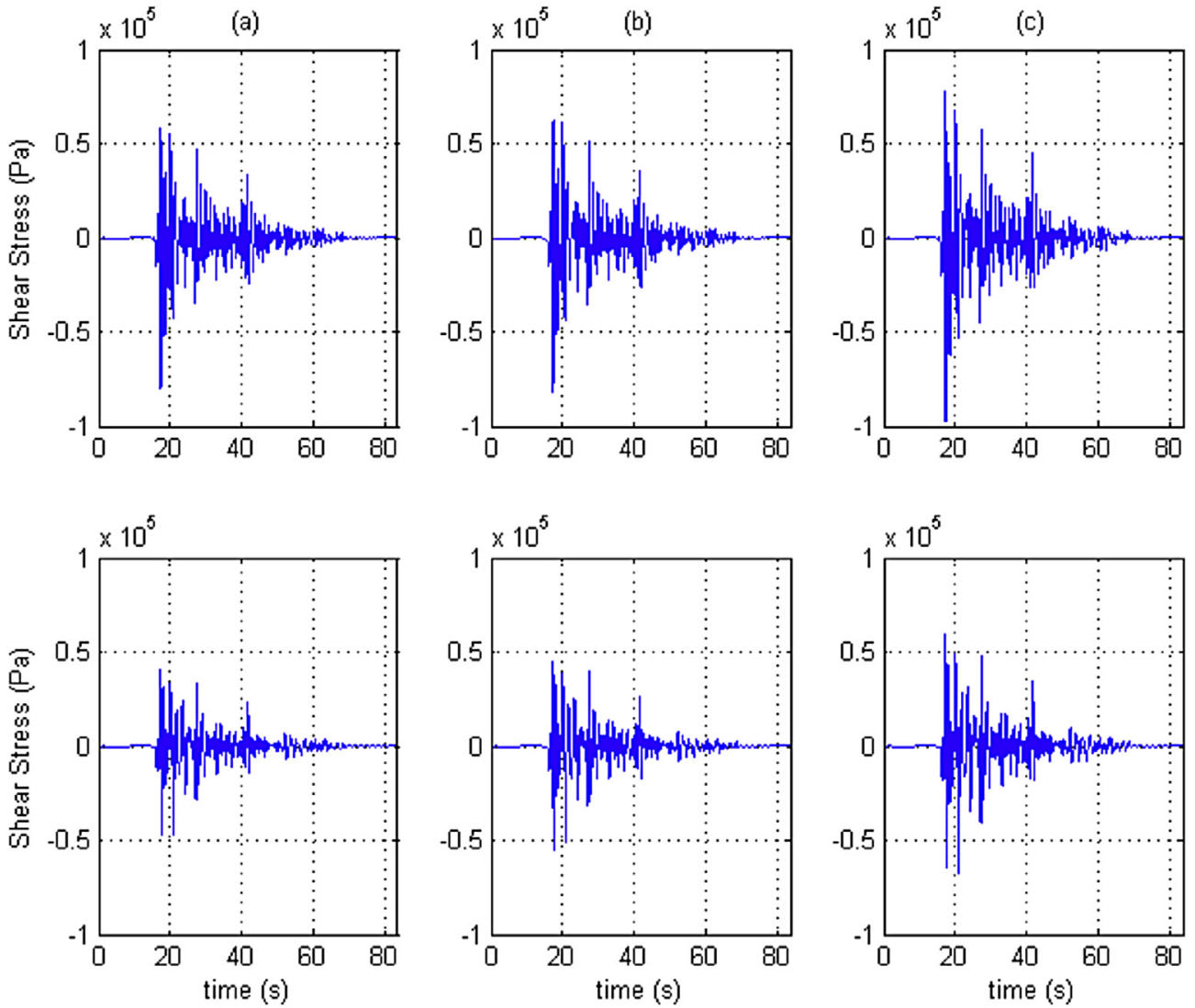

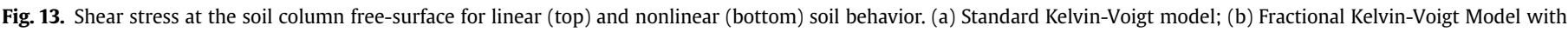

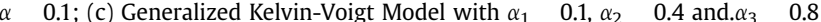



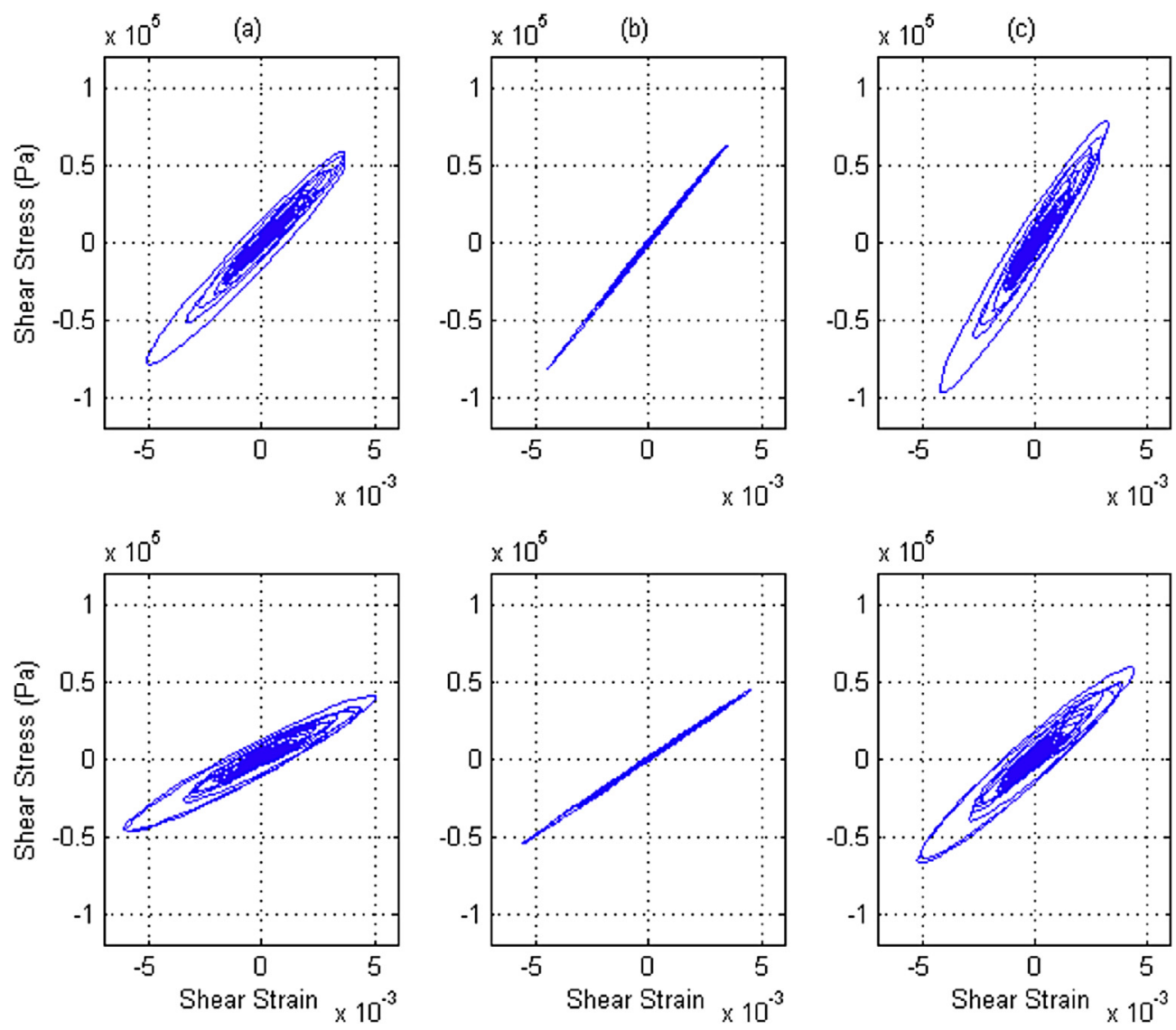

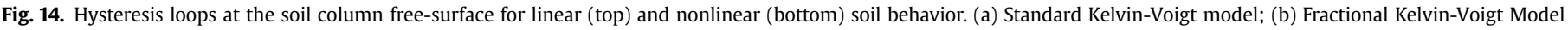
$\begin{array}{lllll}\text { with } \alpha & 0.1 \text {; (c) Generalized Kelvin-Voigt Model with } \alpha_{1} & 0.1, \alpha_{2} & 0.4 \text { and. } \alpha_{3} & 0.8\end{array}$

In the linear case both of them remain constant all along the simulation. The solution was validated by comparing the computed solutions with the ones obtained by using the EERA software in the case of standard damping.

Fig. 9 illustrates the acceleration versus time of the bedrock that constitute the problem loading. Figs. 10-14 show respectively the difference between the linear and nonlinear solutions related to displacement, acceleration, shear strain, shear stress and hysteresis loop, at the surface of the soil deposit. In these figures "Case (a)" refers to the standard Kelvin Voigt model; "Case (b)" to the frac tional Kelvin Voigt model using one fractional coefficient with $\alpha \quad 0.1$ and "Case (c)" the Generalized Fractional Kelvin Voigt model using three fractional coefficients $\alpha_{1} \quad 0.1, \alpha_{2} \quad 0.4$ and $\alpha_{3} \quad$ 0.8. Fractional coefficients are arbitrarily chosen in order to evaluate the effect on that choice in the computed solution. It can be noticed that the consideration of the fractional dashpot and multiple fractional dashpots in Figs. 10-13 has a quite slight impact on the computed solution, however, in Fig. 14 it can be noticed that both fractional dashpot and multiple fractional dashpots have a significant impact on the area of the hysteresis ellipse, leading to a way of finely tuning mechanical behaviors with a reduced number of extra parameters.

\section{Conclusions}

This work analyzed the effects of fractional derivatives in visco elastodynamics. We proved that the use of fractional derivatives for representing the viscous terms offers a large flexibility in the resulting models, higher than the one obtained by using standard derivatives.

For increasing such a flexibility we proposed a generalized Kelvin Voigt model that has only sense when proceeding with fractional derivatives and that allows controlling dissipation as well as the magnitude of deformation and phase angle.

When applied to seismic site response, results prove the higher impact of considering nonlinear versus linear soil behaviors, with respect to the quite reduced differences that the use of fractional damping induces.

Obviously these rich models involve many parameters, the ones associated with the behavior and the ones related to the fractional derivatives. The parametric analysis of all these models require efficient numerical techniques able to simulate complex behaviors. The Proper Generalized Decomposition is the perfect candidate for producing such kind of parametric solutions. Within the PGD framework material parameters and the different derivation powers could be introduced as extra coordinates in the solution procedure. The first simulations are very encouraging and they will reported in future publications.

\section{Appendix A. On fractional derivatives}

There are many books on fractional calculus and fractional dif ferential equations (e.g (Kilbas et al., 2006). (Podlubny, 1999)). We summarize here the main concepts needed to understand the de velopments carried out in this paper.

We start with the formula usually attributed to Cauchy for evaluating the $n$th integration, $n \in \mathbb{N}$, of a function $f(t)$ : 
$J^{n} f(t): \quad \int \cdots \int_{0}^{t} f(\tau) d \tau \quad \frac{1}{\left(\begin{array}{ll}n & 1\end{array}\right) !} \int_{0}^{t}\left(\begin{array}{ll}t & \tau\end{array}\right)^{n-1} f(\tau) d \tau$

This can be rewritten as

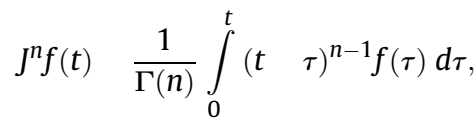

where $\Gamma(n)$ (n 1)! is the gamma function. The latter being in fact defined for any real value $\alpha \in \mathbb{R}$, we can define the fractional integral from

$J^{\alpha} f(t): \frac{1}{\Gamma(\alpha)} \int_{0}^{t}(t \quad \tau)^{\alpha-1} f(\tau) d \tau$.

Now, if we consider the fractional derivative of order $\alpha$, we select an integer $m \in \mathbb{N}$ such that $m \quad 1<\alpha<m$, and it suffices to consider

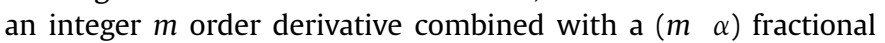
integral. Obviously, we could take the derivative of the integral or the integral of the derivative, resulting in the left and right hand definitions of the fractional derivative usually denoted by $D^{\alpha} f(t)$ and $D_{*}^{\alpha} f(t)$ respectively.

Because these approaches to the fractional derivative began with an expression for the repeated integration of a function, one could consider a similar approach for the derivative. This was the route considered by Grunwald and Letnikov - GL - that defined the so called 'differintegral' that leads to the fractional counterpart of the usual finite differences.

It turns out that the composition of fractional derivatives follows a rule similar to that for standard derivatives. On the other hand, the Fourier transform of a fractional derivative of order $\alpha$ reads $\mathcal{F}(g(t) ; \omega) \quad(i \omega)^{\alpha} \mathcal{G}(\omega)$. This property is particularly useful when addressing harmonic responses as the ones considered in this work.

\section{References}

Bradet, J.P., Ichii, K., Lin, C.H., 2000. EERA: a Computer Program for Equivalent-linear Earthquake Site Response Analyses of Layered Soil Deposits. University of Southern California, Department of Civil Engineering.

Bozorgnia, Y., Bertero, V.V., 2004. Earthquake Engineering: from Engineering Seismology to Performance-based Engineering. CRC press, pp. 415421.

Chinesta, F., Ladeveze, P., Cueto, E., 2011. A short review on model order reduction based on Proper Generalized Decomposition. Arch. Comput. Methods Eng. 18, 395404.

Chinesta, F., Ammar, A., Cueto, E., 2010. Recent advances and new challenges in the use of the Proper Generalized Decomposition for solving multidimensional models. Arch. Comput. Methods Eng. 17/4, 327350.

Chinesta, F., Leygue, A., Bordeu, F., Aguado, J.V., Cueto, E., Gonzalez, D., Alfaro, I. 2013. Parametric PGD based computational vademecum for efficient design, optimization and control. Arch. Comput. Methods Eng. 20/1, 3159.

Chinesta, F., Keunings, R., Leygue, A., 2014. The Proper Generalized Decomposition for Advanced Numerical Simulations. A primer, Springerbriefs, Springer.

Clough, R.W., Penzien, J., 1993. Dynamics of Structures. Civil Engineering Series. McGraw-Hill, New York, NY.

Crandall, S.H., 1970. The role of damping in vibration theory. J. Sound Vib. 11/1, 318.

Germoso, C., Aguado, J.V., Fraile, A., Alarcon, E., Chinesta, F., 2016. Efficient PGDbased dynamic calculation of non-linear soil behavior. Comptes Rendus Acad. Sci. 344, 2441.

Kilbas, A., Srivastava, H.M., Trujillo, J.J., 2006. Theory and Applications of Fractional Differential Equations. Elsevier.

Pecker, A., 1984. Dynamique des sols. Presses de l'Ecole Nationale des Ponts et Chaussées.

Podlubny, I., 1999. Fractional Differential Equations. Academic Press, San Diego.

Quraishi, S., Schroder, C., Mehrmann, V., 2014. Solution of large scale parametric eigenvalue problems arising from brake squeal modeling. In: Proceedings in Applied Mathematics and Mechanics (PAMM), vol. 14. Wiley-VCH Verlag GmbH \& Co. KGaA, Weinheim, pp. 891892.

PB. Schnabel, J. Lysmer and HB. Seed. SHAKE-91: Equivalent linear seismic response analysis of horizontally layered soil deposits. the earthquake engineering online archive NISEE e-Library, http://nisee.berkeley.edu/elibrary/Software/ SHAKE91ZIP.

Seed, H.B., Idriss, L.M. 1970. Soil Moduli and Damping Factors for Dynamic Response Analyses. Report No. EERC 70 10. Earthquake Engineering Research Center; University of California, Berkeley.

Tisseur, F., Meerbergen, K., 2001. The quadratic eigenvalue problem. SIAM Rev. 43 (2), 235286 